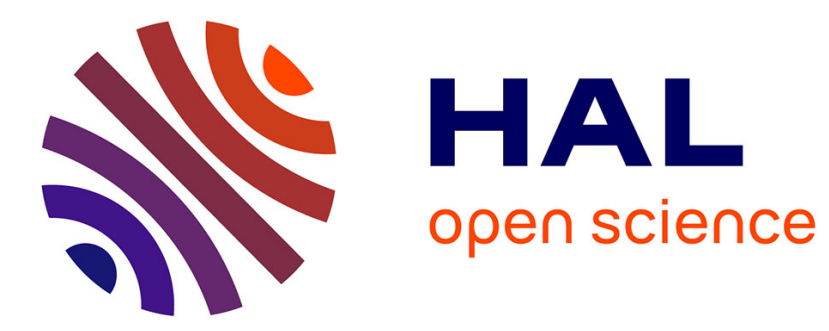

\title{
Spectral approach to finite Reynolds number effects on Kolmogorov's 4/5 law in isotropic turbulence
}

\author{
J. Tchoufag, Pierre Sagaut, Claude Cambon
}

\section{To cite this version:}

J. Tchoufag, Pierre Sagaut, Claude Cambon. Spectral approach to finite Reynolds number effects on Kolmogorov's 4/5 law in isotropic turbulence. Physics of Fluids, 2012, 24, pp.015107. 10.1063/1.3678334. hal-00780160

\section{HAL Id: hal-00780160 \\ https://hal.science/hal-00780160}

Submitted on 8 Apr 2016

HAL is a multi-disciplinary open access archive for the deposit and dissemination of scientific research documents, whether they are published or not. The documents may come from teaching and research institutions in France or abroad, or from public or private research centers.
L'archive ouverte pluridisciplinaire HAL, est destinée au dépôt et à la diffusion de documents scientifiques de niveau recherche, publiés ou non, émanant des établissements d'enseignement et de recherche français ou étrangers, des laboratoires publics ou privés. 


\title{
Spectral approach to finite Reynolds number effects on Kolmogorov's 4/5 law in isotropic turbulence
}

\author{
J. Tchoufag, ${ }^{1}$ P. Sagaut, ${ }^{1}$ and C. Cambon ${ }^{2}$ \\ ${ }^{1}$ Institut Jean Le Rond d'Alembert, UMR 7190, 4 place Jussieu - case 162, \\ Université Pierre et Marie Curie - Paris 6, F-75252 Paris cedex 5, France \\ ${ }^{2}$ Laboratoire de Mécanique des Fluides et d' Acoustique, Ecole Centrale de Lyon, \\ UMR 5509, CNRS, INSA, UCB, 69134 Ecully Cedex, France
}

(Received 11 July 2011; accepted 14 December 2011; published online 31 January 2012)

The Kolmogorov's 4/5 law is often considered as the sole exact relationship of inertial range statistics. Its asymptotic character, however, has been evidenced, investigating the finite Reynolds number (FRN) effect for the third-order structure function $S_{3}(r)$ (e.g., for longitudinal velocity increments with $r$ separation length) using variants of the Kármán-Howarth equation in physical space. Similar semiempirical fits were proposed for the maximum of the normalized structure function, $C_{3}=-\max _{r} S_{3}(r) /(\varepsilon r)$, expressing $C_{3}-4 / 5$ as a power law of the Taylor-based Reynolds number. One of the most complete studies in this domain is by Antonia and Burratini [J. Fluid Mech. 550, 175 (2006)]. Considering that these studies are based on a model for the unsteady second-order structure function $S_{2}(r, t)$, with no explicit model for the third-order structure function itself, we propose to revisit the FRN effect by a spectral approach, in the line of Qian [Phys. Rev. E 55, 337 (1997), Phys. Rev. E 60, 3409 (1999)]. The spectral transfer term $T(k, t)$, from which $S_{3}(r, t)$ is derived by an exact quadrature, is directly calculated by solving the Lin equation for the energy spectrum $E(k, t)$, closed by a standard triadic (or threepoint) theory, here Eddy Damped Quasi Normal Markovian. We show that the best spectral approach to the FRN effect is found by separately investigating the negative (largest scales) and positive (smaller scales) bumps of the transfer term, and not only by looking at the maximum of the spectral flux or $\max _{k} \int_{k}^{\infty} T(p, t) d p \rightarrow \varepsilon$. In the forced case, previous results are well reproduced, with Reynolds numbers as high as $R e_{\lambda}=5000$ to nearly recover the $4 / 5$ value. In the free decay case, the general trend is recovered as well, with an even higher value of $R e_{\lambda}=50000$, but the EDQNM plots are systematically below those in Antonia and Burattini [J. Fluid Mech. 550, 175 (2006)]. This is explained by the sensitivity to initial data for $E(k)$ in solving the Lin equation at moderate Reynolds numbers. Accordingly, an ad hoc initialization yields results consistent with the experimental spectrum measurements of Comte-Bellot and Corrsin [J. Fluid Mech. 48(2), 273 (1971)], from which $S_{3}(r)$ are recalculated. Present results show that the dispersion observed in existing data at low Reynolds number may be due to sensitivity to initial spectrum shape, a feature of the flow which is not under control in most of laboratory experiments. () 2012 American Institute of Physics. [doi:10.1063/1.3678334]

\section{INTRODUCTION}

It is well accepted that the Kolmogorov 4/5 law for the third-order structure function is one of the most important exact equations in turbulence theory. This also reflects that the similarity hypotheses proposed by Kolmogorov (K41) and their subsequent revision (K62) to account for intermittency have had a major impact on turbulence research.

This classical law is expressed as

$$
S_{3}(r)=\left\langle\left(\delta u_{L}\right)^{3}\right\rangle(r)=-\frac{4}{5} \varepsilon r
$$


with $\varepsilon$ the dissipation rate and $\delta u_{L}$ the velocity increment (e.g., longitudinal here) along the separation vector $\boldsymbol{r}$ of modulus $r$. This "exact" law assumes homogeneous and isotropic turbulence, at least in the inertial range, as well as a turbulent dissipation rate which is quasi-independent of viscosity (e.g., Refs. 1 and 2). The aim of our article is not to assess in general the validity of this law, although our study brings new elements, rediscussed in our conclusion, to the jig-saw: Our focus is on very important corrections due to the finite Reynolds number (FRN) effect. Several studies, indeed, faced with the large departure of experimental and numerical results from this law, have demonstrated its asymptotic character, and the possibility to reach it but only at very high Reynolds numbers: Antonia and Burattini (2006) (Ref. 3) recently contributed to a large survey of experimental and numerical results, and gathered various proposals to fit the Taylor-based Reynolds numbers $R_{\lambda}$ dependence, ${ }^{4-8}$ or FRN effect. They separated different behaviors for "forced" and "decay" case and improved existing models at moderate and low Reynolds number in the decay case.

The experimental and numerical results reported by Ref. 3 have been completed by some new plots, which collapse on two curves, presented by Antonia in a summer school held in Cargèse, France, in 2007, reproduced in the book. ${ }^{9}$ The main results are gathered in Fig. 1, thanks to data provided by Antonia (private communication).

In the case of free decay, a typical normalized third-order structure function, or

$$
C_{3}=\max _{r} S_{3}^{*}(r), \quad S_{3}^{*}(r)=\frac{\left\langle\left(\delta u_{L}\right)^{3}\right\rangle(r)}{-\varepsilon r},
$$

approaches the "theoretical" value of $4 / 5$ at Taylor-based Reynolds numbers $R_{\lambda}$ no smaller than $5 \times 10^{4}$. In a "forced" case, the convergence begins to occur at a $R_{\lambda}$ close to $5 \times 10^{3}$.

For the free decay, the simplest way to reproduce these trends is to start from the KármánHowarth equation. A useful variant is the following "full Kolmogorov equation"10

$$
S_{3}(r, t)=-\frac{4}{5} \varepsilon r+6 \nu \frac{\partial S_{2}}{\partial r}-\frac{3}{r^{4}} \int_{0}^{r} r^{\prime 4} \frac{\partial S_{2}}{\partial t} d r^{\prime},
$$

which expresses $S_{3}$ in terms of the unsteady second-order structure function $S_{2}(r, t)$. Only isotropy and homogeneity are required, and it is obvious that the departure from the $4 / 5$ law is mediated by explicit viscous and unsteady terms, which can be easily modeled using conventional scalings for $S_{2}$. The main results of these analyses are reported in Table I for the normalized third-order structure function and in Table II for its maximum value, i.e., the $C_{3}$ parameter. A model by Lundgren ${ }^{7}$ yields

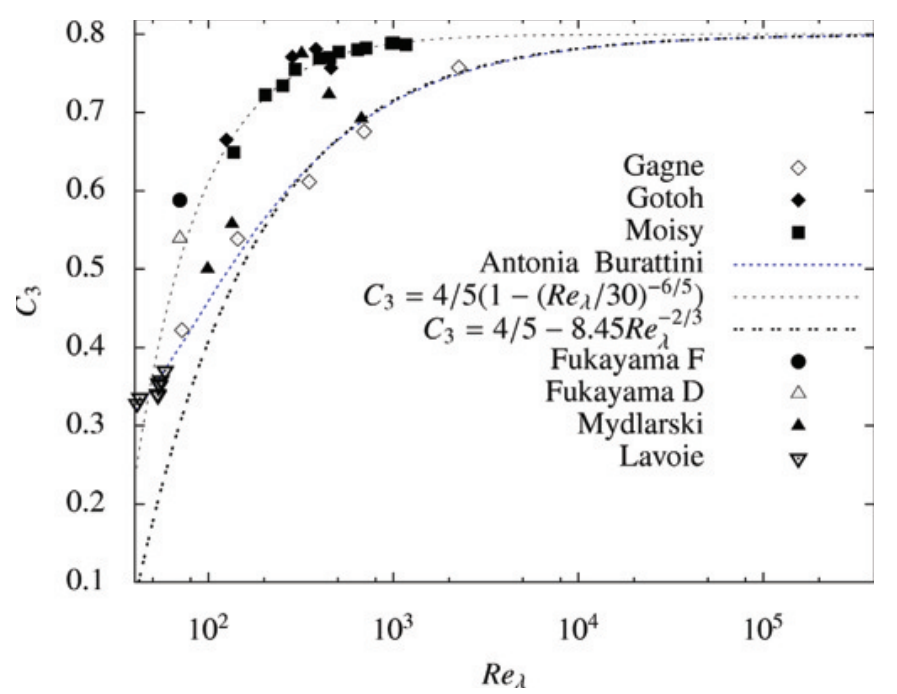

FIG. 1. (Color online) Adapted from Antonia and Burattini (2006). Black symbols are for "forced" turbulence cases, from experiments or DNS, white symbols from "free decay" cases. Semi-empirical fits are in dashed lines. 
TABLE I. Empirical laws for $S_{3}^{*}(r)$.

\begin{tabular}{|c|c|c|}
\hline Source & $S_{3}^{*}(r)$ & Remarks \\
\hline Qian (1999) & $\frac{4}{5}-C_{1}\left(\frac{r}{L}\right)^{m}-C_{2}\left(\frac{r}{\eta}\right)^{-4 / 3}$ & $\begin{array}{l}\text { Decaying turbulence, } \\
C 1>0, C_{2}=5.26 K_{0}\end{array}$ \\
\hline Moisy (1999) & $\frac{4}{5}-8\left(\frac{r}{\eta}\right)^{-4 / 3}-\frac{2}{7} R_{\lambda}^{-3}\left(\frac{r}{\eta}\right)^{2}$ & - \\
\hline Lindborg (1999) & $\frac{4}{5}-2\left(C_{\star} R_{\lambda}^{-1}\left(\frac{r}{\eta}\right)^{2 / 3}+4\left(\frac{r}{\eta}\right)^{-4 / 3}\right)$ & $C_{\star}=\frac{4 \sqrt{15} 1.6}{17}$ \\
\hline Lundgren (2002) & $\frac{4}{5}-\frac{3.34}{\sqrt{15}}\left(\frac{r}{L}\right)^{2 / 3}-8\left(\frac{r}{\eta}\right)^{-4 / 3}$ & - \\
\hline Lundgren (2003) & $\frac{4}{5}\left(1-R_{\lambda}^{-2 / 3}\left(2.678 C_{2}\left(\frac{r}{\lambda}\right)^{2 / 3}+2.029 C_{2}\left(\frac{r}{\lambda}\right)^{-4 / 3}\right)\right)$ & Forced turbulence, $C_{2} \simeq 2$ \\
\hline Lundgren (2003) & $\frac{4}{5}\left(1-R_{\lambda}^{-2 / 3}\left(2.678\left(\frac{2}{3} \frac{1+n}{n}\right) C_{2}\left(\frac{r}{\lambda}\right)^{2 / 3}+2.029 C_{2}\left(\frac{r}{\lambda}\right)^{-4 / 3}\right)\right)$ & Decaying turbulence, $q^{2} \propto t^{-n}$ \\
\hline
\end{tabular}

$$
C_{3}=\frac{4}{5}-8.45 R_{\lambda}^{-2 / 3}
$$

An even simpler model for $S_{2}(r, t)$, to be re-injected in Eq. (3), is used by Lindborg ${ }^{4}$ by means of Kolmogorov scaling and $k-\varepsilon$ modeling for unsteady $\varepsilon$, yielding a fit similar to Eq. (4). In fact, quasi identity of both scalings in Refs. 4 and 7 comes from the high-Reynolds Kolmogorov estimate of $S_{2}$,

$$
S_{2}=C_{2}(\varepsilon r)^{2 / 3}
$$

using the same constant $C_{2}=2$. Finally, following Ref. 11, the approach in Ref. 3 modifies Eq. (3) by replacing the elapsed time by a streamwise spatial coordinate and the normalized $S_{2}$ by a modeled closure. This model, derived from Eq. (2.8) in Ref. 3, is reported in Fig. 1: it coincides with the Lundgen model (Ref. 7, Eq. (4)) for $R_{\lambda} \geq 200$, but significantly differs at smaller $R_{\lambda}$ with an inflexion branch towards higher $C_{3}$, in very good agreement with plots from grid-turbulence experiments. $^{12,13}$

In the forced case, a fit proposed by by Moisy et al. (1999) (Ref. 6), or

$$
C_{3}=\frac{4}{5}\left(1-\left(\frac{R_{\lambda}}{R_{\lambda_{0}}}\right)^{-6 / 5}\right),
$$

is also reported on Fig. 1. It was first checked along data from turbulence in low temperature helium gas, with an optimal value $R_{\lambda_{0}} \sim 30$. On the other hand, the proposal by Lundgren for "linearly forced turbulence" is significantly below the plots from physical and experimental data and, therefore, not used here.

Note that the flow cases reported in Fig. 1 are so different that there is no reason whatsoever to expect universal asymptotics, such as Eqs. (4) and (5) towards Kolmogorov universal laws. In that respect, the really interesting result may be that flows too different to be a priori comparable fall on the same or adjacent curves in Figures 1 and 3. Just mention that "Moisy" holds for a von Karman flow, and even with co-rotating discs, so that turbulence production is fed by a complex recirculating mean flow; flows generated by active grids are sometimes considered as forced; Gagne's data gather results from grid-turbulence and jet. Therefore, arises the question: to what extent can the FRN effect be disconnected from effects of anisotropy and even inhomogeneity (obviously considering homogeneity as local, in a dedicated range of scales, whose range depends on the Reynolds number)? This question and the general support of this work by existing experiments, or by new ones in progress, is rediscussed in conclusion. 
TABLE II. Empirical laws for $C_{3}$

\begin{tabular}{|c|c|c|}
\hline Source & $C_{3}(r)$ & Remarks \\
\hline Qian (1999) & $\frac{4}{5}-C_{\delta} R_{\lambda}^{-\mu}, \mu=\frac{6 m}{3 m+4}$ & $\begin{aligned} \text { Decay: } \mu= & 2 / 3, \text { hom. shear: } 2 / 3 \leq \mu \leq 1, \\
& \text { inhom. shear: } \mu \geq 1\end{aligned}$ \\
\hline Lindborg (1999) & $\frac{4}{5}-6 C_{\star}^{2 / 3} R_{\lambda}^{-2 / 3}$ & - \\
\hline Moisy (1999) & $\frac{4}{5}\left(1-\left(\frac{R_{\lambda}}{R_{\lambda_{0}}}\right)^{-6 / 5}\right)$ & $R_{\lambda 0} \sim 30$ \\
\hline Lundgren (2002) & $\frac{4}{5}-8.45 R_{\lambda}^{-2 / 3}, \mu=\frac{6 m}{3 m+4}$ & $\begin{aligned} & \text { Decay: } \mu=2 / 3 \text {, hom. shear: } 2 / 3 \leq \mu \leq 1, \\
& \text { inhom. shear: } \mu \geq 1\end{aligned}$ \\
\hline Lundgren (2003) & $\frac{4}{5}\left(1-2.678 R_{\lambda}^{-2 / 3} C_{2}\left(\frac{2.029}{2.678}\right)^{1 / 3}\right)$ & Forced turbulence, $C_{2} \simeq 2$ \\
\hline Lundgren (2003) & $\frac{4}{5}\left(1-2.678 R_{\lambda}^{-2 / 3} C_{2}\left(\frac{2.029}{2.678}\right)^{1 / 3}\left(\frac{2(1+n)}{3 n}\right)^{2 / 3}\right)$ & Decay $q^{2} \propto t^{-n}$ \\
\hline
\end{tabular}

The possibility to extend our study to anisotropic flows, subjected to body forces, with related production of energy and/or alteration of cascade is one of our motivation to apply a spectral approach. The scalar Kármán-Howarth equation and its variant Eq. (3), indeed, cannot be systematically generalized to flows with forcing and "production." A forced version of the KármánHowarth equation is used by Frisch $^{2}$ to maintain stationarity, but only stochastic isotropic forcing is considered. On the other hand, a generalized spectral approach can account for physical forcing better than the sole modification of the Kármán-Howarth equation, as shown further, in our
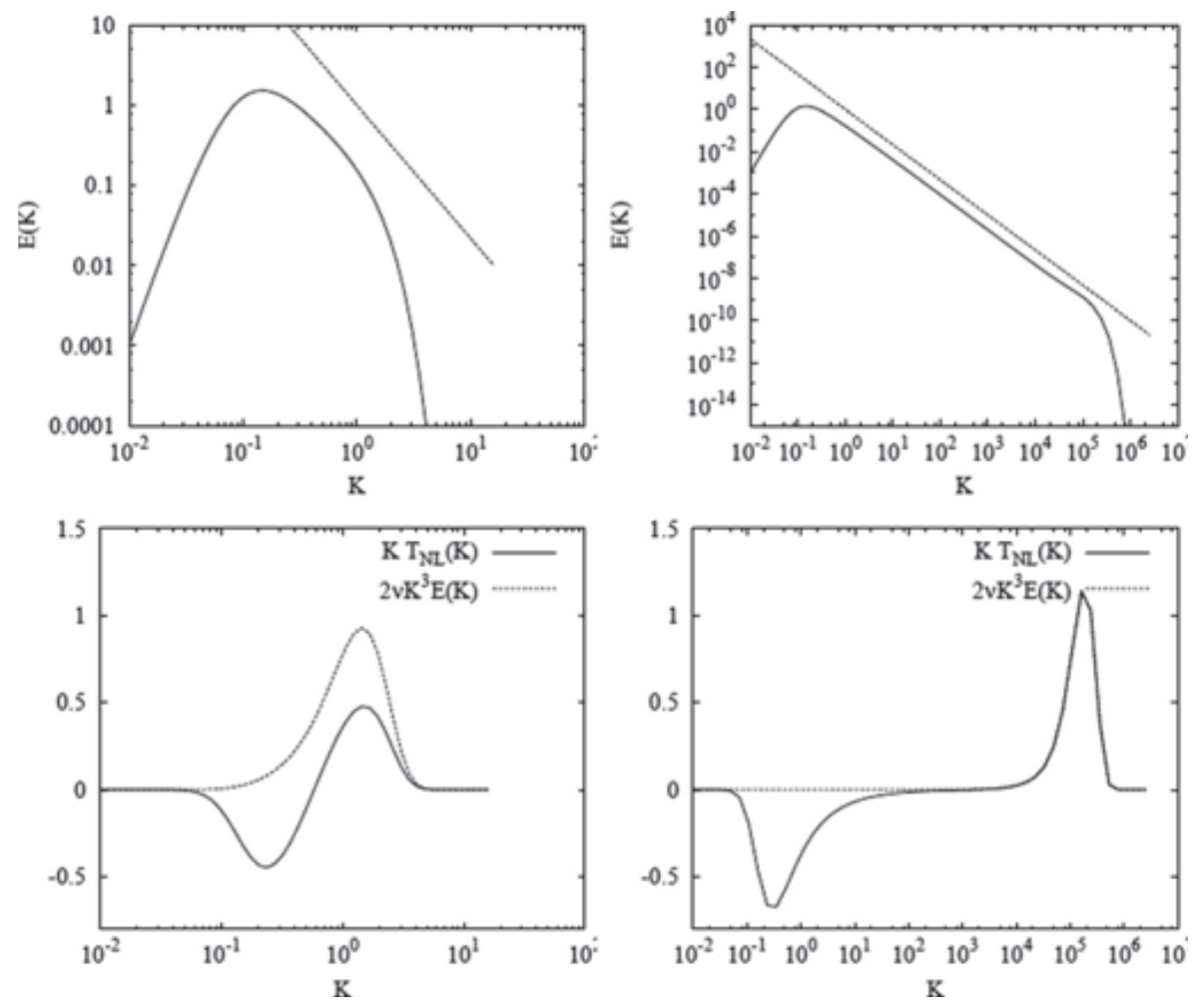

FIG. 2. Typical spectral results: Left, $R_{\lambda}=30$ and right, $R_{\lambda}=10^{5}$. Top, energy spectra; the straight line gives the $-5 / 3$ slope. Bottom, solid line, energy transfer $T(k)$; dashed line, dissipation spectrum, $2 \nu k^{2} E(k)$; both spectral transfer and dissipation spectrum are multiplied by $k$ in order to display the zero integral property of the first one in semi-logarithmic coordinates. From EDQNM runs by Wouter Bos in Sagaut and Cambon (2008) (Ref. 9) with permission of CUP. 


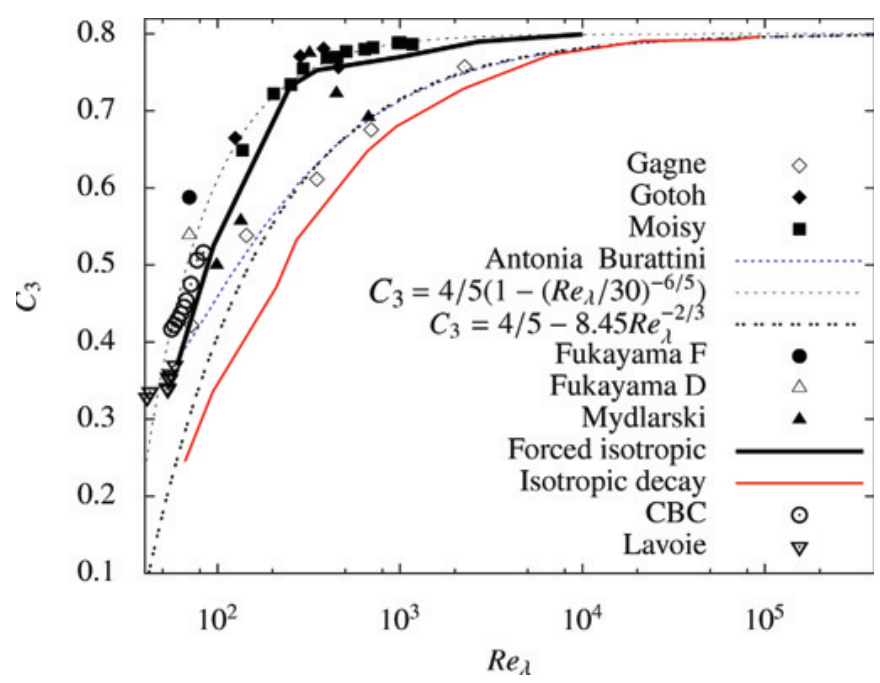

FIG. 3. (Color online) Plots of Kolmogorov's function $C_{3}$ versus Reynolds number, as in Fig. 1, and in addition: $C_{3}$ values reconstructed from EDQNM simulations in both forced (upper full line) and freely decaying case (lower full line); plots reconstructed by EDQNM from Comte-Bellot and Corrsin data (CBC), white circles.

discussion of Eddy Damped Quasi Normal Markovian (EDQNM), in Secs. III and IV. Going back to isotropic turbulence, the Lin equation can be used, as proposed by Qian, ${ }^{5,14}$ as a counterpart of the Eq. (3). In addition, it is possible to directly relate the spectral transfer $T(k, t)$ to the dynamics of third-order correlations at three points, in the line of the triadic closures of Kraichnan and Orszag. These approaches, therefore, tackle the problem of cascade at the level of three-point third-order dynamics. This is more complex than deriving a model for $S_{3}(r, t)$ from a model for $S_{2}(r, t)$, as proposed by Ref. 3 and other authors using Eq. (3), but this offers a deeper investigation of cascade, possibly modified by anisotropic physical forcings. The analysis of Qian was just mentioned for his scaling by the authors cited above, but his deeper underlying spectral analysis was almost ignored.

We, therefore, propose to revisit and extend Qian's approach in this article. We shall try to first answer the question: What is the equivalent of the 4/5-law, when derived from the Lin equation, and what is the spectral counterpart of the FRN effect?

In the second part, we shall perform a quantitative study by numerically solving the Lin equation, closed by a standard approach such as EDQNM. This procedure can take into account arbitrary unstationarity and arbitrary value of the Reynolds number, really from 0 to infinity, without assuming a priori a Kolmogorov law for $E(k, t)$ (as it is done for $S_{2}(r, t)$ ). This type of closure is a good candidate for a quantitative spectral analysis: it has been checked at low Reynolds numbers using the data from Comte-Bellot and Corrsin (CBC) (1971), ${ }^{15}$ e.g., in Ref. 16; it compares very well with truncated Euler equations at infinite Reynolds number ${ }^{17,18}$ and provides a sensible basis for investigating the decay exponent with uncertainties. ${ }^{20}$

The Lin equation (Lin 1949 (Ref. 21)) governs the energy spectrum $E(k, t)$ as

$$
\frac{\partial}{\partial t} E(k, t)+2 \nu k^{2} E(k, t)=T(k, t)
$$

and the spectral transfer term $T(k, t)$ is mediated by third-order velocity correlations. The thirdorder structure function can be derived in terms of $T(k, t)$ by an exact quadrature. ${ }^{14}$

To the first question of the spectral equivalence of the 4/5 law, the answer is not so simple, in spite of the analyses by Frisch ${ }^{2}$ and especially Qian. It is obvious that Kolmogorov laws at second order are really similar, with

$$
S_{2}(r)=C_{2}(\varepsilon r)^{2 / 3}, \quad E(k)=K_{0} \varepsilon^{2 / 3} k^{-5 / 3},
$$

in the inertial range, and significant ranges of $-5 / 3$ power law are found for $E(k)$, with a very low dispersion of the $K_{0}$ constant, ${ }^{22}$ even at moderate Reynolds number. A departure from the $-5 / 3$ 
exponent was studied by Gamard and George (1999) (Ref. 23) but it was shown to reflect a FRN effect and not a correction due to an hypothetical internal intermittency. This viewpoint is also supported by Lundgren (2002) (Ref. 24) using a theoretical approach and by McComb (2009) (Ref. 25). We prefer not enter the debate on intermittency here, restricting our focus to the FRN effect.

Analogies between approaches carried out in physical and in Fourier space are not so obvious at third order: Dropping both the explicit unsteady and viscous terms in the Lin equation, following the same procedure as in Eq. (3), yields the unsatisfactory and too simple result $T(k, t)=0$ : $T=0$ is not obtained over a whole inertial range, but only for a single wavenumber $k^{*}$; We recover here what was presented by McComb (2008) as a paradox. ${ }^{26}$ At least a conventional counterpart of $C_{3}=4 / 5$ in Eq. (2) is the maximum of the energy flux, defined by

$$
\Pi(k, t)=\int_{k}^{\infty} T(p, t) d p
$$

tending towards

$$
\max _{k} \Pi(k)=\varepsilon
$$

(e.g., Direct Numerical Simulation (DNS) results presented by Kaneda at the Cargese 2007 Summerschool from Ref. 27, see also Ref. 28). We propose here to refine the approach to 4/5 Kolmogorov law in reconciling the viewpoints of Sagaut and Cambon (2008), "The 4/5 law is as exact as its counterpart in Fourier space $\int_{k^{*}}^{\infty} T(k) \mathrm{d} k=\varepsilon$ " and of McComb, "A paradox and its resolution:" both approaches dissociate the ranges of negative and positive transfers, without assuming an exact large $T=0$ plateau.

For the quantitative approach, we shall show the importance of initial data for $E(k)$ in the correct evaluation of plots for free decay derived from solving the Lin equation. Recovering $C_{3}$ from the spectral data of Comte-Bellot and Corrsin ${ }^{29}$ is a crucial point in this article as well.

This article is organized as follows. The qualitative approach to 4/5 law based on the Lin equation, and related FRN effect is given in Sec. II, using the exact equation to relate the third order structure function to the spectral transfer term. Equations for its closure by EDQNM are recalled in Sec. III, with a numerical approach. Section IV is devoted to final discussion of results, conclusions, and perspectives.

\section{QUALITATIVE APPROACH TO THE 4/5 LAW FROM THE SPECTRAL TRANSFER TERM}

\section{A. Exact equations for two-point third-order velocity correlations and related spectral transfer terms}

The general tensor $Q_{i l m}(\boldsymbol{r})=\left\langle u_{i}(\boldsymbol{x}) u_{l}(\boldsymbol{x}) u_{m}(\boldsymbol{x}+\boldsymbol{r})\right\rangle$ can be expressed in terms of $u^{\prime 3} K(r)=r^{-3} Q_{i l m} r_{i} r_{l} r_{m}$ only as

$$
Q_{l m i}(\boldsymbol{r})=u^{\prime 3}\left[\frac{1}{2}\left(K-r \frac{\partial K}{\partial r}\right) \frac{r_{l} r_{m} r_{i}}{r^{3}}+\frac{1}{4}\left(2 \frac{K}{r}+\frac{\partial K}{\partial r}\right)\left(r_{i} \delta_{l m}+r_{m} \delta_{i l}\right)-\frac{1}{2} K \frac{r_{l}}{r} \delta_{i m}\right],
$$

using homogeneity, isotropy $-Q_{l m i}$ is a tensorial isotropic function of $\boldsymbol{r} / \boldsymbol{r}$-and incompressibility (see, e.g., Eq. (6.141) in Ref. 30 in which $\boldsymbol{x}-\boldsymbol{r}$ and $\boldsymbol{x}$ are used, whereas our convention is to use $\boldsymbol{x}$ and $\boldsymbol{x}+\boldsymbol{r}$ following Ref. 31).

Similarly, the corresponding spectral tensor $T_{i j m}$, or the 3D inverse Fourier transform of $Q_{i j m}$, using

$$
Q_{i j m}(\boldsymbol{r})=\iiint T_{i j m}(\boldsymbol{k}) \exp (\imath \boldsymbol{k} \cdot \boldsymbol{r}) d^{3} \boldsymbol{k},
$$

is expressed as

$$
T_{i j m}(\boldsymbol{k})=-\imath \frac{T(k)}{8 \pi k^{3}}\left[\frac{k_{i}}{k}\left(\delta_{j m}-\frac{k_{j} k_{m}}{k^{2}}\right)+\frac{k_{j}}{k}\left(\delta_{i m}-\frac{k_{i} k_{m}}{k^{2}}\right)\right],
$$

in terms of the transfer term, the same as in Eq. (6), 


$$
T(k)=4 \pi k^{2} l k_{i} T_{i j j}(\boldsymbol{k}) .
$$

Note that the simplest way to find Eq. (11) is not to use the term-to-term Fourier transform of Eq. (9), but to directly express $T_{i j m}$ as an isotropic tensorial function of $\boldsymbol{k} / \boldsymbol{k}$, using the algebraic counterpart of incompressibility $k_{m} T_{i j m}=0$; one recovers exactly in the previous equation the Kraichnan's projection operator, which is applied to the basic nonlinear term of Navier-Stokes equation in Fourier space.

Replacing Eq. (11) into the direct 3D Fourier transform which links $Q_{i j m}(\boldsymbol{r})$ to $T_{i j m}(\boldsymbol{k})$, any cubic velocity correlation can be expressed in terms of $T$ by a simple quadrature. This can be shown by using a polar-spherical frame of reference for $\boldsymbol{k}$ in Eq. (10), with polar axis aligned with $\boldsymbol{r}$, at fixed $\boldsymbol{r}$. The new set of variables for $\boldsymbol{k}$ is its modulus $k$, the cosine of the polar angle, or $x=\boldsymbol{k} \cdot \boldsymbol{r} /(\mathrm{kr})$, and the azimutal angle $\phi_{k}$. In terms of those, the integral Eq. (10) becomes

$$
Q_{i j m}(\boldsymbol{r})=\int_{0}^{\infty} k^{2} \mathrm{~d} k\left(\int_{-1}^{1} \mathrm{~d} x \exp (\imath k r x)\left(\int_{0}^{2 \pi} T_{i j m}(\boldsymbol{k}) \mathrm{d} \phi_{k}\right)\right),
$$

so that $u^{\prime 3} K(r)$ is expressed as

$$
u^{\prime 3} K(r)=\int_{0}^{\infty} k^{2} \mathrm{~d} k\left(\int_{-1}^{1} \mathrm{~d} x \exp (\imath k r x)\left(\int_{0}^{2 \pi} T_{i j m}(\boldsymbol{k}) \frac{r_{i} r_{l} r_{m}}{r^{3}} \mathrm{~d} \phi_{k}\right)\right) .
$$

From Eq. (11) is found

$$
T_{i j m} \frac{r_{i} r_{j} r_{m}}{r^{3}}=-l \frac{T(k)}{4 \pi k^{3}} x\left(1-x^{2}\right)
$$

so that the integral on $\phi_{k}$ reduces to a simple multiplication by $2 \pi$, whereas the $x$-part is explicit, and

$$
u^{\prime 3} K(r)=2 r \int_{0}^{\infty} \underbrace{\left[\frac{1}{4 l} \int_{-1}^{1} x\left(1-x^{2}\right) \frac{\exp (\imath k r x)}{k r} \mathrm{~d} x\right]}_{g_{5}(k r)} T(k) \mathrm{d} k,
$$

is finally expressed by a single $k$-integral, in which the weighting function $g_{5}$ in terms of $k r$ is analytically calculated as

$$
g_{5}(k r)=\frac{3(\sin k r-k r \cos k r)-(k r)^{2} \sin k r}{(k r)^{5}} .
$$

The third-order structure function readily derives from Eq. (14) using

$$
S_{3}(r)=\left\langle\left(\delta u_{L}\right)^{3}\right\rangle(r)=6 u^{13} K(r)=12 r \int_{0}^{\infty} g_{5}(k r) T(k) \mathrm{d} k,
$$

so that it involves the same weighting function $g_{5}(k r)$ given by Eq. (15).

The function $g_{5}(k r)$ is nonsingular at $k r=0$ and has the limiting behaviors

$$
\begin{aligned}
g_{5}(k r) \rightarrow \frac{1}{15}, \quad k r & \rightarrow 0, \\
g_{5}(k r) \sim-\frac{\sin k r}{(k r)^{3}}, \quad k r & \rightarrow \infty .
\end{aligned}
$$

If the separation is much smaller than the Kolmogorov scale, $r \ll \eta, k r$ is small over the entire range of the spectrum. Expanding $g(k r)$ for small argument, the first term contributes zero to the final integral in Eq. (14), and we need to go one order further:

$$
g_{5}(k r) \sim \frac{1}{15}-\frac{1}{210}(k r)^{2}
$$


to find

$$
u^{\prime 3} K(r) \sim-\frac{1}{105} r^{3} \int_{0}^{\infty} k^{2} T(k) \mathrm{d} k
$$

hence,

$$
\left\langle\left(\delta u_{L}\right)^{3}\right\rangle \sim-\frac{2}{35} r^{3} \int_{0}^{\infty} k^{2} T(k) \mathrm{d} k .
$$

The $r^{3}$ dependence is to be expected at sub-Kolmogorov scales since the velocity field is smooth when viewed at such scales. The integral is dominated by the dissipative range, as we might also expect. The integral $\int_{0}^{\infty} k^{2} T(k) d k$ is exactly the nonlinear vortex stretching term $\left\langle u_{i, j} \omega_{i} \omega_{j}\right\rangle$ mediated by nonlinearity in the equation governing the enstrophy $(1 / 2)\left\langle\omega_{i} \omega_{i}\right\rangle=\int_{0}^{\infty} k^{2} E(k) d k=\varepsilon /(2 \nu)$.

At high enough Reynolds numbers such that there is an inertial range, there are two distinct and compensating contributions to

$$
\int_{0}^{\infty} T(k) \mathrm{d} k=0 .
$$

The first comes from the large scales, $k=O\left(L^{-1}\right)$, and equals $-\varepsilon$, while the second arises from the region of positive transfer, and equals $\varepsilon$. If $r$ lies in the inertial range, the presence of $g_{5}(k r)$ in Eq. (16) effectively removes the contribution of positive transfer, close to the dissipative contribution thanks to the decay of $g_{5}(k r)$ like $\frac{\sin k r}{k r}(k r)^{-2}$ at large argument, whereas $k r$ is small when $k=O\left(L^{-1}\right)$ and we can replace $g_{5}(k r)$ by $1 / 15$ in that range. Thus, the final integral in Eq. (14) should take the value $-\langle\epsilon\rangle / 15$ in the inertial range, leading to

$$
u^{\prime 3} K(r)=-\frac{2}{15} \varepsilon r
$$

and hence the Kolmogorov 4/5 law (1).

\section{B. From low to high Reynolds qualitative analysis, isotropic decay case}

A qualitative approach to the 4/5 law is first illustrated by Fig. 2 as follows. At low Reynolds number, the transfer term consists of two successive bumps, a negative one (drain of energy from large scales) and a positive one (transfer towards smaller scales), which have the same area and are separated by a single point of zero transfer at $k=k^{*}$. The area of the negative bump, or energy drain from largest scales $\varepsilon_{f}$,

$$
\varepsilon_{f}=-\int_{0}^{k^{*}} T(k, t) \mathrm{d} k
$$

is much smaller than the area, $\varepsilon$, of the dissipation spectrum $2 \nu k^{2} E(k, t)$, or

$$
\varepsilon_{f}=-\int_{0}^{k^{*}} T(k) \mathrm{d} k=\int_{k^{*}}^{\infty} T(k) \mathrm{d} k \ll \int_{0}^{\infty} 2 \nu k^{2} E(k) \mathrm{d} k=\varepsilon,
$$

so that the effective integral resulting from the constant value $1 / 15$ of the weighting function $g_{5}(\mathrm{kr})$ in Eq. (14) yields a third-order structure function significantly smaller (in absolute value) than its asymptotic value $\frac{4}{5} \varepsilon r$. Note that the energy drain $\varepsilon_{f}$ from largest scales corresponds to the "spectral consumption" of Lumley; ${ }^{32}$ in the case of spectral equilibrium, it provides a better evaluation of the dissipation rate than the classical small-scale viscous term, as rediscussed by Llor. ${ }^{33}$

Only at very high Reynolds number, the two bumps of opposite sign are separated by an apparent plateau (see also Ref. 26), and the positive energy transfer, present at small scales, coincides scale-by-scale with the dissipation spectrum. It is, therefore, obvious from the analysis of 
Subsection II A that the 4/5 Kolmogorov law is recovered, but with a separate validity of stationarity of $E(k)$, at smallest scales, and removal of explicit dissipation spectrum, at largest scales.

- At largest scales (smallest $k$ 's), the dissipation spectrum can be neglected, so that $\partial E(k, t) /$ $\partial t=T(k, t)$; by integrating from 0 to $k^{*}$, one finds the large-scale balance $\mathrm{d} \mathcal{K} / \mathrm{d} t=-\varepsilon_{f}$ for the kinetic energy $\mathcal{K}$. According to the behavior of $g_{5}$ at small argument, one recovers $\int_{0}^{k^{*}} g_{5}(k r) T(k) d k=-\frac{1}{15} \varepsilon_{f}$.

- At smallest scales (largest $k$ 's), the instationarity term in the Lin equation can be neglected, but not the dissipation spectrum, so that $2 \nu k^{2} E(k)=T(k)$; by integrating from $k^{*}$ to $\infty$, one recovers the spectral balance $\varepsilon=\varepsilon_{f}$. The convergence to zero of the integral, mentioned in Sec. II A, $\int_{k^{*}}^{\infty} g_{5}(k r) T(k) \mathrm{d} k$ in this domain, completes the proof of the 4/5 law.

- The range of the quasi-plateau is much more reduced than the $-5 / 3$ range for the energy spectrum: Almost 7 decades for the $-5 / 3$ slope versus 3 decades for $T \sim 0$ at $R_{\lambda}=10^{5}$ in Fig. 2 . In addition, a refined analysis shows that the zero transfer is found at a single point (a single $k=k^{*}$ value) at any Reynolds number, even if a quasi-plateau ( $T$ almost zero but not exactly zero) appears at largest $R_{\lambda}$, in the free-decay case. This structure of the transfer term near $k=k^{*}$ was recently presented as a paradox by McComb; ${ }^{26}$ this is rediscussed in Sec. III in both free-decay and forced cases.

\section{Are additional assumptions needed for asymptotic convergence?}

This preliminary analysis shows the interest of separating in Fourier space the two domains of sink and source for the energy transfer, with common area $\varepsilon_{f}$. It is confirmed that the FRN effect and the related departure from the 4/5 law are linked to the spectral imbalance $\varepsilon_{f}<\varepsilon$. Before applying a rather complex closure technique, it is possible to mimic what was done in physical space ${ }^{4}$ by reinjecting the Kolmogorov evaluation of $E(k, t)$ in Eq. (7) with time-dependent $\varepsilon=\varepsilon(t)$. This yields immediately the single value of $k^{*}$ in term of the inverse of the longitudinal Taylor length scale

$$
k^{*}=\sqrt{\frac{20}{3} \frac{n+1}{n}} / \lambda_{f},
$$

classically defined from $\varepsilon=20 \nu \mathcal{K} / \lambda_{f}^{2}$, where $-n$ is the decay exponent of the kinetic energy, in agreement with $\mathrm{d} \ln \varepsilon / \mathrm{d} \ln \mathcal{K}=(n+1) / n$. (or optimal value of the $k-\varepsilon$ "constant" as well). This simple procedure gives an evaluation of the positive slope of $T(k)$ at $k=k^{*}$, which tends to zero as a power of $1 / R_{\lambda}$. On the other hand, the shape of $T(k)$ is completely missed by this oversimplified approach outside the vicinity of its zero value, with divergence in both small and large $k$ 's, respectively, as $k^{-5 / 3}$ and $k^{1 / 3}$.

More generally, it is perhaps useful to investigate to which extent our "proof of the 4/5 law" in this section needs some additional assumptions, in the asymptotic limit of really vanishing viscosity, following Refs. 1 and 2. As suggested by Christos Vassilicos (private communication), the existence of both an outer lengtscale $\left(\ell_{o}\right)$ and an inner one $\left(\ell_{i}\right)$ is implicitely assumed, with a condition of very large separation in the vanishing viscosity limit. The outer scale $\ell_{o}$, or $\ell_{o}(t)$ considering the unsteady decay case, exists (independently of viscosity) such that for any $k^{*} \ell_{o} \gg 1 \int_{0}^{k *} E(k, t) \mathrm{d} k \rightarrow \int_{0}^{\infty} E(k, t) \mathrm{d} k=\mathcal{K}(t)$, with the same relationship for the time derivative yielding $d \mathcal{K} / d t$. A second implicit assumption is that the ratio $2 \nu \int_{0}^{k^{*}} k^{2} E(k, t) \mathrm{d} k$ to $\varepsilon$ must tend to zero in the same limit. The existence of $\ell_{o}(t)$ and $\ell_{i}(t)$ is thus inferred to ensure that $\int_{0}^{k^{*}} T(k, t) \mathrm{d} k \rightarrow-\varepsilon$, and $T(k, t) \rightarrow 0$, in the range $\ell_{0}^{-1} \ll k^{*} \ll l_{i}^{-1}$, with $\ell_{i} \leq \lambda$. Last, this analysis can be seen as to evaluate $S_{3}(r, t) /(12 r)=\int_{0}^{\infty} g_{5}(k r) T(k, t) \mathrm{d} k$ by splitting the spectral domain into three ranges, defined as $\ell_{0}^{-1} \ll k_{0} \ll k_{i} \ll \ell_{i}^{-1}$ and by considering $r$ into $k_{i}^{-1} \ll r \ll k_{o}^{-1}$. With regards to these ranges, the following relations hold in the $\nu \rightarrow 0$ limit: $\int_{0}^{k_{o}} g_{5}(k r) T(k, t) \mathrm{d} k \rightarrow-\frac{\varepsilon}{15}, \int_{k_{i}}^{k_{o}} g_{5}(k r) T(k, t) \mathrm{d} k \sim 0$, and $\int_{k_{i}}^{\infty} g_{5}(k r) T(k, t) \mathrm{d} k \rightarrow 0$.

Finally, we emphasize that these implicit assumptions are used along only with the Lin equation and the exact integral relationship between $T$ and $S_{3}$. It is supported qualitatively by Fig. 2 


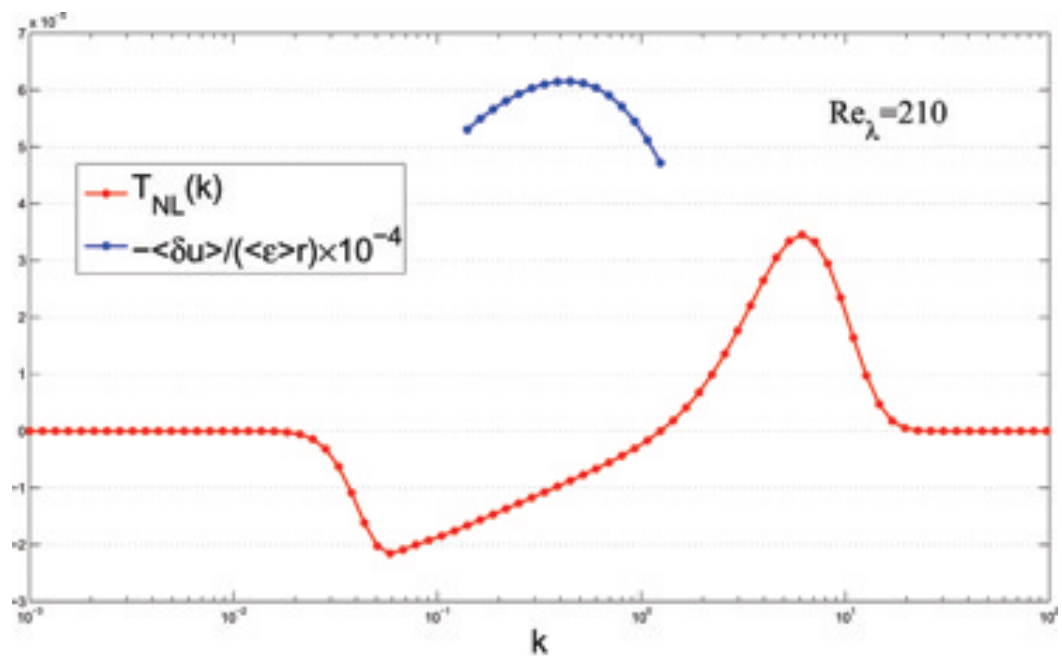

(a)

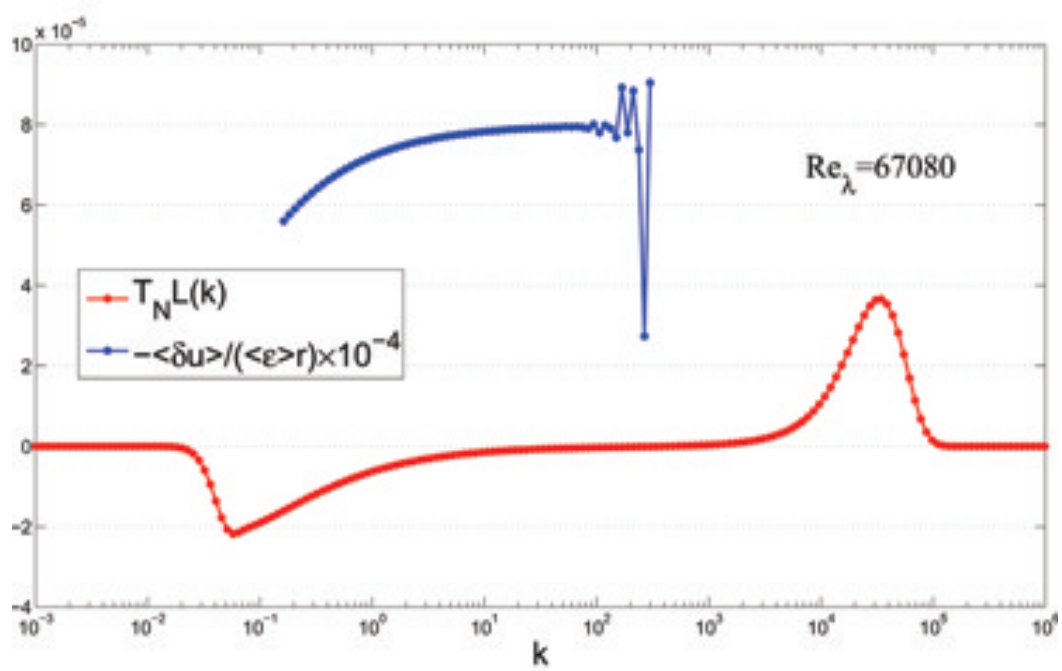

(b)

FIG. 4. (Color online) Lower curve: Transfer function $T(k)$ at low Reynolds number $\left(R_{\lambda}=210\right)$ and high Reynolds number $\left(R_{\lambda}=67080\right)$ in the free decay case. Upper curve: value of the normalized function $S_{3}^{*}(r)$ versus $r=1 / k$, where $S_{3}^{*}(r)$ is defined according to Eq. (29).

and quantitatively by Figs. 4 and 5 in Sec. III. However, the above discussion does not refine the closure of $T(k, t)$ in terms of $E(k, t)$, this issue being left for a subsequent paper.

\section{EDQNM PROCEDURE AND RESULTS}

It is possible to derive the time-history of the third-order structure function from the one of $T(k, t)$, using Eqs. (15) and (16), by solving the Lin equation with various initial data. For this purpose, $T(k)$ is closed in terms of the energy spectrum by a conventional EDQNM model. The detailed derivation is not recalled for the sake of brevity (see Refs. 34 and 35 and turbulence books), and only the main steps and the final equations, with specific adjustments, are given in the following.

First, it is perhaps useful to recall how EDQNM derives from a "triadic" approach, based on the dynamics of three-point third-order correlations. At order three, the dynamical equation that governs triple correlations has to be taken at three points, in order to account for exact corrections from pressure fluctuations in connection with incompressibility constraint. A complete approach in physical space is not available, in spite of advanced equations and discussions by Batchelor, 


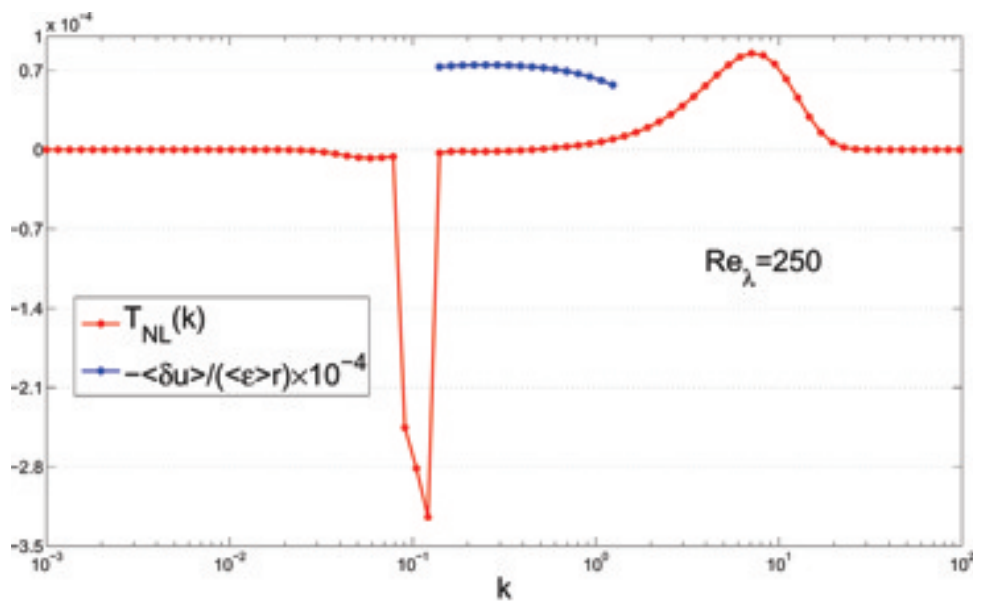

(a)

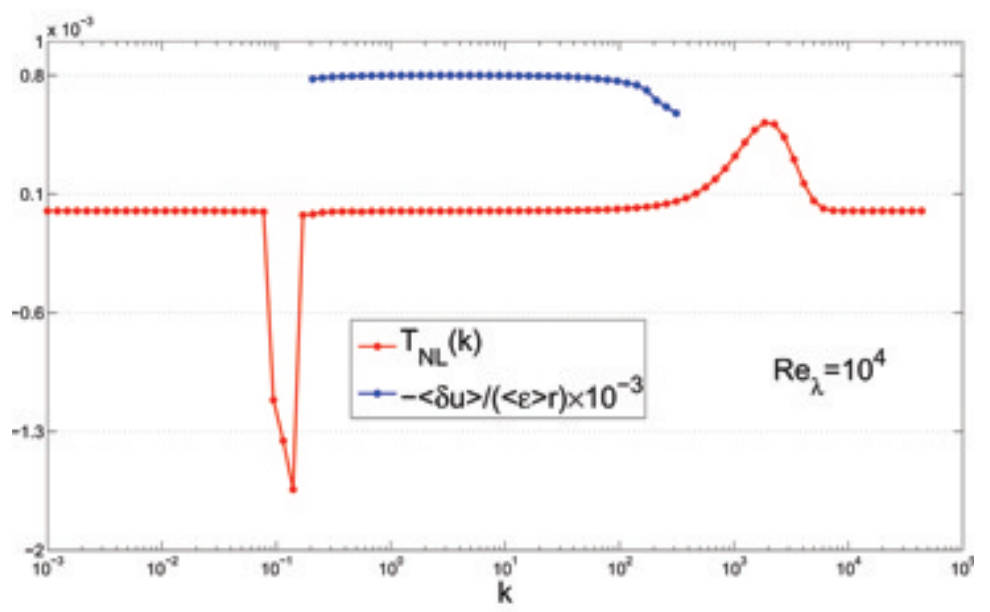

(b)

FIG. 5. (Color online) Lower curve: Transfer function $T(k)$ at low Reynolds number (top, $R_{\lambda}=250$ ) and high Reynolds number (bottom, $R_{\lambda}=10000$ ) in the forced case. Upper curve: value of the normalized function $S_{3}^{*}(r)$ versus $r=1 / k$, where $S_{3}^{*}(r)$ is defined according to Eq. (29).

Craya (1958, full Ph. D. report, in French) and in Davidson's ${ }^{36}$ book. The counterpart of the equation which governs triple correlations at three points is only given in Fourier space, under a form in which pressure contributions are exactly solved, by means of projection operators. The relevant tensor is $S_{i j j m}$ defined by

$$
\left\langle\hat{u}_{i}(\boldsymbol{q}) \hat{u}_{j}(\boldsymbol{k}) \hat{u}_{m}(\boldsymbol{p})\right\rangle=S_{i j m}(\boldsymbol{k}, \boldsymbol{p}) \delta^{3}(\boldsymbol{k}+\boldsymbol{p}+\boldsymbol{q}),
$$

so that

$$
T_{i j m}(\boldsymbol{k})=\iiint S_{i m j}(\boldsymbol{k}, \boldsymbol{p}) d^{3} \boldsymbol{p},
$$

and it is governed by the dynamical equation

$$
\left(\frac{\partial}{\partial t}+\nu\left(k^{2}+p^{2}+q^{2}\right)\right) S_{i j m}(\boldsymbol{k}, \boldsymbol{p}, t)=T_{i j m}^{I V}(\boldsymbol{k}, \boldsymbol{p}, t),
$$

in which $\boldsymbol{q}=-\boldsymbol{k}-\boldsymbol{p}$ and $T^{I V}$ holds for contribution of fourth-order correlations at three points, with their exact corrections for pressure terms. This level of description offers the best opportunity to introduce a robust closure, such as the (Eddy damped quasi normal) EDQN one 


$$
T_{i j m}^{I V}(\boldsymbol{k}, \boldsymbol{p}, t)=T_{i j m}^{Q N}(\boldsymbol{k}, \boldsymbol{p}, t)-(\eta(k, t)+\eta(p, t)+\eta(q, t)) S_{i j m}(\boldsymbol{k}, \boldsymbol{p}, t) .
$$

The dominant term is the quasi-normal (QN) one, $T^{Q N}$, which amounts to rewrite $T^{I V}$ in terms of a sum of products of second-order correlation tensors, as for a normal law. The correction term, with the Eddy-damping term $\eta(k, t)$ given below, therefore, corresponds to the contribution of fourth-order cumulants, which are modeled as a relaxation term to reinforce the dissipative effect in Eq. (23), following heuristic arguments.

The final expression for the transfer term utilizes the EDQN closure above, in addition to simplifications given by isotropy for the second-order spectral tensor involved in $T_{i m j}^{Q N}$. The last simplification, called Markovianization (M) amounts to truncate the proper time-memory of three-point triple correlations (22) in Eq. (23). The following compact expression of $T(k, t)$ in terms of $E$ is straightforward via the quadratic nonlocal (in $k$-space) relationship, resulting from a QN closure consistent with solenoidal properties, but instantaneous thanks to a Markovianization (M), and corrected by an Eddy damping (ED)

$$
T(k, t)=\iint_{\Delta_{k}} \theta_{k p q}\left(x y+z^{3}\right) E(q, t)\left(E(p, t) p k^{2}-E(k, t) p^{3}\right) \frac{d p d q}{p q},
$$

with

$$
\theta_{k p q}=\left(\nu\left(k^{2}+p^{2}+q^{2}\right)+\eta(k, t)+\eta(p, t)+\eta(q, t)\right)^{-1},
$$

in which $\Delta_{k}$ is the domain of $(p, q)$ at fixed $k$, so that $k, p$, and $q$ are the lengths of the sides of a triangle, $x y+z^{3}$ accounts for exact solenoidal properties, $x, y$, and $z$ being the cosines of the internal angles of the triangle of sides $k, p, q$, and $\eta$ is the semi-empirical ED term introduced by Orszag (1970). It was shown that the use of

$$
\eta(k, t) \sim \varepsilon^{1 / 3} k^{2 / 3},
$$

yielded a satisfactory behavior when solving numerically the Lin equation, with the establishment of a Kolmogorov inertial range for $E(k, t)$. We prefer using here the variant by Ref. 37, or

$$
\eta(k, t)=A\left(\int_{0}^{k} p^{2} E(p, t) d p\right)^{1 / 2},
$$

which amounts to choosing $\eta$ as the inverse of a Corrsin (or Batchelor as well) time scale, without specifying a power law as in Eq. (27). The constant $A$ is related here to a well accepted value of the Kolmogorov constant $K_{0}, K_{0} \sim 2.76 A^{2 / 3}$ as in Ref. 38, but a less constraining choice is given by Ref. 14, who presents another interpretation of $\eta$. In addition, the latter analysis explains why any conventional closure of the form Eq. (25), even if predicting different values of $K_{0}$, is well suited for approaching the finite Reynolds effect (see also Ref. 19 or Local Energy Theory (LET) theor ${ }^{39}$ for a version with self-adapted $\eta$ ).

We now investigate how the EDQNM results match the data illustrated in Fig. 2 in both forced turbulence and freely decaying turbulence, considering a wide range of Reynolds numbers.

In the forced turbulence case, the Taylor-scale-based Reynolds number $R_{\lambda}$ is kept constant using a classical forcing term which acts at very large scales. The analysis is performed once a steady equilibrium energy spectrum is obtained. It has been checked that the shape of the forcing term has a negligible influence on the transfer function $T(k)$.

In the freely decaying case, a high-Reynolds initial condition with a von Kármán spectrum is first prescribed, even at low $R_{\lambda}$. The simulation is then performed until a self-similar solution with a time-independent decay exponent is reached. Once such a state is reached, the spectrum is rescaled in order to enforce the targeted Reynolds number, and the simulation is continued until a new self-similar solution is obtained. The transfer term $T(k)$ is stored at this point. It is carefully checked that self-similar states are obtained before storing the data (see Ref. 20 for a detailed analysis of EDQNM prediction of decay exponents during self-similar decay). 
For plotting a single point for a given value of $R_{\lambda}$ from EDQNM, we follow the same procedure as Antonia and Burattini (2006) to compute the optimal value of the Kolmogorov parameter $C_{3}$, i.e.,

$$
C_{3}=\max _{r} S_{3}^{*}(r), \quad S_{3}^{*}(r)=\frac{\left\langle\left(\delta u_{L}\right)^{3}\right\rangle(r)}{-\varepsilon r}=12 \frac{\int_{0}^{\infty} g_{5}(k r) T(k) \mathrm{d} k}{-2 \nu \int_{0}^{\infty} p^{2} E(p) d p}
$$

New EDQNM results are compared in Fig. 3 to data already shown in Fig. 1. All trends reported by previous authors are recovered here, but significant differences are observed in the free decay case, especially at low Reynolds numbers, for which experimental data also exhibit a significant dispersion. The "forced" EDQNM curve is in good agreement with "Moisy's" fit, whereas the "decay" EDQNM curve is significantly below the corresponding plots in Ref. 3. In order to discuss these results, we shall successively check the sensitivities with respect to $r$ in Eq. (29) and to the initial energy spectrum.

\section{A. Sensitivity with respect to the choice of $r$}

We now further investigate the differences that exist between the forced and freely decaying case. Samples of $T(k)$ computed in these two configurations, at both high and low Reynolds numbers, are displayed in Figs. 4 and 5, along with the evolution of $C_{3}(r)$ versus $r=1 / k$. A careful examination of the transfer function $T(k)$ yields an explanation of the difference between forced and free decay cases. In the latter, a real plateau with $T(k)=0$ is almost never observed, even at high Reynolds numbers at which viscous effects can be neglected over a wide range of scales. At $R_{\lambda}=67080$, it is observed that $T(k)=0$ is approached over a single decade only. The reason is that the unsteady term $\partial E(k, t) / \partial t$ is non-negligible over the entire inertial range. In contrast, for the forced case, $\partial E(k, t) / \partial t=0$ at all scales, leading to the existence of a real plateau with $T(k)=0$. At $R_{\lambda}=10$ 000, a 3-decades wide plateau is observed.

This has also an effect on the sensitivity of estimated $S_{3}^{*}(r)$ versus $r$. Defining the Kolmogorov parameter, $C_{3}$ as the maximum of $S_{3}^{*}(r)$ rises the problem of the accuracy of the description of $T(k)$. Present results show that, in the free decay case, the maximum is approached on a very narrow spectral band for $1 / r$, showing that $T(k)$ must be very accurately described. In the same way, if the Kolmogorov constant is computed using direct measures of the third-order structure function $\left\langle\left(\delta u_{L}\right)^{3}\right\rangle(r)$, this quantity must be very accurately discretized versus $r$. Evaluating $\max _{r} S_{3}^{*}(r)$ in the forced case is much less difficult in the forced case, thanks to the existence of a wide plateau, even at medium Reynolds numbers. Wiggles observed on $S_{3}^{*}(r)$ at small values of $r$ in the very-high Reynolds number freely decaying case are due to round-off errors when computing the integral Eq. (29), illustrating the accuracy problem that arises when very large scale ratios are considered.

\section{B. Sensitivity with respect to $E(k)$ initialization}

The experiment by Comte-Bellot and Corrsin of grid turbulence is probably the best documented in the literature, with the special care devoted to approach isotropy. From the measurements, it was possible to derive spectra at three sections and even transfer terms by interpolating $E(k, t)$ to derive $\partial E / \partial t$. A first use of the Lin equation closed by EDQNM, initializing $E(k)$ at the first cross-section $x / M=42$, yielded excellent agreement in recovering both energy spectra and transfer terms at the two downstream sections $(x / M=91$ and $x / M=171)$ and the transfer term related to the first section as well. ${ }^{16}$ This suggests to redo the calculation and reconstruct the thirdorder structure function from the transfer term, using Eq. (16), because the third-order structure function was not directly measured in the experiment. It appears that the plots for $C_{3}$ derived from this new calculation are now significantly above the ones with von Kármán spectrum initialization, and even suggest a curvature with upward concavity, as in the low-Reynolds part of the AntoniaBurattini curve.

Looking at Fig. 3, it is clear that one can modify the parameter $C_{3}$ by a factor of about two by just changing the initial spectrum in the numerical EDQNM procedure. The use of a von Kármán spectrum $^{40}$ in the standard initialization (red curve) overpredicts the dissipation rate at the 
denominator. The new data labeled $\mathrm{CBC}$, obtained using experimentally measured spectra at initial time, are a bit above the Antonia-Burattini model, but this is not inconsistent with the fact that the best fits for "forced" and "decay" cases cross over at a relatively low Reynolds number, with $C_{3}$ possibly larger in the decay case than in the forced case in this domain. The large scattering of both experimental or DNS data illustrates well the possible uncertainty in $C_{3}$ from other methods: The reader is referred to Ref. 3 for more details on these results.

\section{CONCLUDING REMARKS}

A full investigation of the departure and eventual approach to Kolmogorov's 4/5 law based on the EDQNM closure has been presented, addressing both Reynolds number dependence and forcing effects. It is shown that Lin's equation for the kinetic energy spectrum $E(k, t)$, with a classical closure for $T(k, t)$, contains the full physics of the problem, since all trends reported in previous works dealing with experimental or DNS data are recovered. The previously reported FRN effect is recovered and extended to Reynolds numbers large-enough to reach the asymptotic- $4 / 5$ value. Important conclusions, which are consistent with findings by previous authors, are that Reynolds numbers as high as $R_{\lambda}=5000$ and $R_{\lambda}=50000$ are, respectively, needed to nearly recover the $-4 / 5$ value in the forced and freely decaying case.

In the past decade, this problem was mainly investigated by looking at the Kármán-Howarth equation and especially at its integrated version in Eq. (3). As illustrated by Ref. 3, with references therein, there exists a huge literature in this context, with modeling $S_{2}(r, t)$, in contrast with the little number of studies based on the direct approach to the spectral transfer term $T(k, t)$.

Considering the free-decay case at $R_{\lambda} \geq 200$, the problem is apparently solved, or at least quantified, by scaling laws in $R_{\lambda}^{-2 / 3}$, but attempts to model $C_{3}$ at lowest Reynolds numbers are often questionable without a deeper investigation. One of our most salient results is the reconstruction of $S_{3}$ and $C_{3}$ using the spectral data from $\mathrm{CBC},{ }^{15}$ with evidence of the crucial importance of an appropriate initial energy spectrum. Another result deals with the sensitivity of the computation of the $C_{3}$ parameter, given the $r$ domain retained in Eq. (29), which is observed to be high in the free decay case.

In spite of the apparent analogy between the Lin equation, in Fourier space, and the KármánHowarth equation, in physical space, there is no simple match between the results around the $4 / 5$ law that can be derived in both spaces. On the one hand, a direct approach to $T(k, t)$, using a triadic third-order closure technique, is much more complicated than a physical space approach, which models only $S_{2}(r, t)$ in the variant of the K-H equation, but not $S_{3}(r, t)$ itself. In this sense, these modeling approaches are two-point second-order; whereas the classical closure theories address the third-order correlations at three points, or for triads in Fourier space, and not only at two points, as specified in Eqs. (22)-(24). On the other hand, the spectral approach does not prescribe a priori the Kolmogorov scalings, and includes much more information, allowing very important properties from basic Navier-Stokes equations to be preserved: detailed conservation of energy and helicity, triad by triad, in three-dimensional space, solenoidal property and related removal of pressure for individual velocity modes, not to mention a tractable Langevin equation for each realization.

In the free-decay case, it is clear that the simultaneous cancellation of unsteadyness and dissipation, which yields the $4 / 5$ law in the Kolmogorov equation, only gives $T(k)=0$. This result is disappointing because a plateau with $T(k)=0$ is almost never observed, and $\partial E(k) / \partial t$ is not negligible over a very wide range of scales (see also the paradox pointed out by Ref. 26.) Instead of looking at the total flux term in Eq. (8) and the convergence of its maximum towards $\varepsilon$, it is more informative to dissociate the negative and positive areas of the transfer term, with the large-scale energy drain $\varepsilon_{f}$ defined from the former zone and the spectral imbalance $\varepsilon-\varepsilon_{f}$ defined from the latter one. It is confirmed that the $4 / 5$ law is recovered in the limit of spectral balance, with separate valididity of unsteadyness, only at smallest scales, and removal of dissipation spectrum, only at largest scales. Nevertheless, one can point out that unsteadyness at the smallest scales, with $T(k)=2 \nu k^{2} E(k)$, approached in Fig. 2 at $R_{\lambda}=10^{5}$, is never strictly verified in all the large $k$ 's domain, in our present runs, even until $R_{\lambda}=67000$. The spectral approach confirms that the $4 / 5$ law is better expressed using $\varepsilon_{f}$ instead of $\varepsilon$, as $S_{3}=-4 / 5 \varepsilon_{f} r$, even if $\varepsilon_{f}$ and $\varepsilon$ eventually coincide at very high Reynolds number. This derives 
from $\int_{0}^{k^{*}} g_{5}(k r) T(k) d k \rightarrow-1 / 15 \varepsilon_{f}$ and from $\int_{k^{*}}^{\infty} g_{5}(k r) T(k) d k \rightarrow 0$, with no need for an explicit assumption on the dissipation rate, and only well-known properties of $T(k)$.

In the forced case, our EDQNM results are not far from the fit $C_{3}-4 / 5 \sim R_{\lambda}^{-6 / 5}$, in close connection with theoretical considerations ${ }^{5}$ recovering Moisy's result. ${ }^{6}$ In this case, a large plateau is really obtained for $T(k)=0$ and sensitivity of the computation of $C_{3}$ to both the $r$ domain and initialization of the energy spectrum is dramatically reduced.

The 4/5 Kolmogorov's law, which is nothing but a simplified version of the Kármán-Howarth equation, holds for isotropic turbulence only. In the present paper, strictly isotropic forcing was used, in a consistent way. Most experimental data used to validate the asymptotic $-4 / 5$ law come from anisotropic shear flows, as illustrated by data used in Fig. 1, and seem to support the concept of "local" homogeneity and isotropy, i.e., limited to an inertial sub-range. In addition to existing experiments, with their drawbacks, one can expect better isotropy and higher Reynolds number from present or future experiments, such as in Ref. 41.

On the other hand, experimental evidence does exist of a departure from the $4 / 5$ law, more dramatic than a simple FRN effect, ${ }^{5}$ not to mention some recent extensions of this study to axisymmetric, rotating or stably stratified turbulence. The influence of physical anisotropic forcing should be investigated in the future, e.g., considering homogeneous anisotropic turbulent flows. It is clear that the fully tensorial, with full wavevectors relationship in Eq. (24) is valid for arbitrary anisotropic turbulence. This is particularly true for the quasi-normal term, or $T_{i j m}^{Q N}$, which can be exactly expressed in terms of the second order spectral tensor $\hat{R}_{i j}\left(\boldsymbol{k}^{\prime}, t\right) \sim\left\langle\hat{u}_{i}\left(-\boldsymbol{k}^{\prime}\right) \hat{u}_{j}\left(\boldsymbol{k}^{\prime}\right)\right\rangle$, without assuming isotropy. Even if an anisotropic form of the fourth-order cumulant relaxation term is possible, it is better to first incorporate in the closure method the "true" linear terms responsible for the anisotropisation. As illustrated in Ref. 9 and in references therein (see Ref. 42 for a new recent application), we have developed for a long time an original procedure for inverting the anisotropic "true" linear operators in equations for cubic moments. This allows us to base the closure on a zeroth-order Kraichnan's response function, which is actually the RDT (rapid distortion theory) exact linear Green's function. In this sense, we generalize EDQNM, but also we incorporate more general concepts coming from Kraichnan's direct interaction approximation (DIA), but in bypassing the strenuous procedure of coupled equations for the Kraichnan's response tensor and the two-time second-order spectral tensor. Note that our procedure can easily incorporate the RDT for cubic correlations, as well as the asymptotic limit of "weak" wave-turbulence theory, when three-wave resonance is permitted by the dispersion law.

The physical meaning of a 4/5 law, given a strong anisotropy in the inertial range, must be revisited in flows without energy production, subjected to rotation, stable stratification, and MHD Alfvénic turbulence, considering that Lin equations can be generalized to them as well as closure theories ranging from weakly nonlinear wave-turbulence theory to anisotropic EDQNM. There is no production of kinetic energy in the first case of rotating turbulence and no production of total (kinetic + potential or kinetic + magnetic) in the other two cases.

\section{ACKNOWLEDGMENTS}

We are grateful to Robert Antonia for providing data files on Fig. 1 and relevant advice, to Christophe Bailly for providing unpublished data from Comte-Bellot and Corrsin (1971) and for reworking them, and to Alexandre Pieri for technical assistance. Significant improvements were suggested by Antoine Llor, David McComb, and Christos Vassilicos. Finally, a part of this work benefitted from a close collaboration with Julian Scott, Fabien Godeferd, and Luminita Danaila, supported by the ANISO Contract No. ANR-88-BLAN-0076.

\footnotetext{
${ }^{1}$ A. S. Monin, A. M. Yaglom, and J. L. Lumley, Statistical Fluid Mechanics (MIT, Cambridge, MA, 1971), Vol. 1.

${ }^{2}$ U. Frisch, Turbulence: The Legacy of A. N. Kolmogorov (Cambridge University Press, Cambridge, 1995).

${ }^{3}$ R. A. Antonia and P. Burattini, "Approach to the 4/5 law in homogeneous isotropic turbulence," J. Fluid Mech. 550, 175 (2006).

${ }^{4}$ E. Lindborg, "Correction to the four-fifths laws due to variation of the dissipation," Phys. Fluids 11, 510 (1999).

${ }^{5}$ J. Qian, "Slow decay of the finite Reynolds number effect of turbulence," Phys. Rev. E 60, 3409 (1999).

${ }^{6}$ F. Moisy, P. Tabeling, and H. Wilaime, "Kolmogorov equation in a fully developed turbulence experiment," Phys. Rev. E 82(20), 3994 (1999).

${ }^{7}$ T. S. Lundgren, "Linearly forced turbulence," CTR Annual Research Briefs (2003).
} 
${ }^{8}$ Y. Gagne, B. Castaing, C. Baudet, and Y. Malecot, "Reynolds dependence of third-order structure functions," Phys. Fluids 16(2), 482 (2004).

${ }^{9}$ P. Sagaut and C. Cambon, Homogeneous Turbulence Dynamics (Cambridge University Press, Cambridge, 2008).

${ }^{10}$ L. D. Landau and E. M. Lifschitz, Course on Theoretical Physics: Fluid Mechanics, (MIR, Moscow, 1989).

${ }^{11}$ L. Danaila, F. Anselmet, T. Zhou, and R. A. Antonia, “A generalization of Yaglom's equation which accounts for the large-scale forcing in heated decaying turbulence," J. Fluid Mech. 391, 359 (1999).

${ }^{12}$ T. Zhou and R. A. Antonia, "Reynolds number dependence of the small-scale structure of grid turbulence," J. Fluid Mech. 406, 81 (2000).

${ }^{13}$ P. Lavoie, P. Burattini, L. Djenidi, and R. A. Antonia, "Effect of initial conditions on decaying grid turbulence at low $r_{\lambda}, "$ Exp. Fluids 39, 865 (2005).

${ }^{14}$ J. Qian, "Inertial range and the finite reynolds effect of turbulence," Phys. Rev. E 55, 337 (1997).

${ }^{15}$ G. Comte-Bellot and S. Corrsin, "Simple Eulerian time correlation of full-and narrow-band velocity signals in gridgenerated 'isotropic' turbulence," J. Fluid Mech. 48(2), 273 (1971).

${ }^{16}$ C. Cambon, D. Jeandel, and J. Mathieu, "Spectral modelling of homogeneous non-isotropic turbulence,” J. Fluid Mech. 104, 247 (1981).

${ }^{17}$ C. Chichowlas, P. Bonati, F. Debbash, and M. Brachet, "Effective dissipation and turbulence in spectrally truncated Euler flows," Phys. Rev. Lett. 95, 264502 (2005).

${ }^{18}$ W. J. T. Bos and J.-P. Bertoglio, "Dynamics of spectrally truncated inviscid turbulence," Phys. Fluids 18, 071701 (2006).

${ }^{19}$ W. J. T. Bos and J.-P. Bertoglio, "A single-point two-time closure based on fluid particle displacements," Phys. Fluids 18, 031706 (2006).

${ }^{20}$ M. Meldi, P. Sagaut, and D. Lucor, “A stochastic view of isotropic turbulence decay,” J. Fluid Mech. 668, 351 (2011).

${ }^{21}$ T. von Kármán and C. C. Lin, "On the concept of similarity in the theory of isotropic turbulence," Rev. Mod. Phys. 21(3), 516 (1949).

${ }^{22}$ K. R. Sreenivasan, "On the universality of the Kolmogorov constant," Phys. Fluids 7, 2778 (1995).

${ }^{23}$ S. Gamard and W. K. George, "Reynolds number dependence of energy spectra in the overlap region of isotropic turbulence," Flow, Turbul. Combust. 63, 443 (1999).

${ }^{24}$ T. S. Lundgren, "Kolmogorov two-thirds law by matched asymptotic expansion," Phys. Fluids 14, 638 (2002).

${ }^{25} \mathrm{D}$. McComb, "Scale invariance and the inertial-range spectrum in three-dimensional, stationary, isotropic turbulence," J. Phys. A: Math. Theor. 42, 125501 (2009).

${ }^{26}$ D. McComb, "Scale invariance in three-dimensional isotropic turbulence: A paradox and its resolution," J. Phys. A: Math. Theor. 41, 075501 (2008).

${ }^{27}$ T. Aoyama, T. Ishihara, Y. Kaneda, M. Yokokawa, K. Itakura, and A. Uno, "Statistics of energy transfer in highresolution direct numerical simulation of turbulence in a periodic box," J. Phys. Soc. Jpn. 74, 3202 (2005).

${ }^{28}$ T. Gotoh, D. Fukayama, and T. Nakano, "Velocity field statistics in homogeneous steady turbulence obtained using highresolution direct numerical simulation," Phys. Fluids 14(3), 1065 (2002).

${ }^{29}$ G. Comte-Bellot and S. Corrsin, "The use of a contraction to improve the isotropy of grid-generated turbulence," J. Fluid Mech. 25, 657 (1966).

${ }^{30}$ J. Mathieu and J. F. Scott, Turbulent Flows: An Introduction (Cambridge University Press, Cambridge, 2000).

${ }^{31}$ G. K. Batchelor, The Theory of Homogeneous Turbulence (Cambridge University Press, Cambridge, 1953).

${ }^{32}$ J. L. Lumley, "Some comments on turbulence," Phys. Fluid A 4, 203 (1992).

${ }^{33}$ A. Llor, "Langevin equation of big structure dynamics in turbulence: Landau's invariant in the decay of homogeneous isotropic turbulence," Eur. J. Mech. B/Fluids, 30, 480 (2011).

${ }^{34}$ S. A. Orszag, "Analytical theories of turbulence," J. Fluid Mech. 41, 363 (1970).

${ }^{35}$ C. E. Leith, “Atmospheric predictability and two-dimensional turbulence,” J. Atmos. Sci. 28, 145 (1971).

${ }^{36}$ P. A. Davidson, Turbulence (Oxford University Press, Oxford, 2004).

${ }^{37}$ A. Fouquet, M. Lesieur, J.-C. André, and C. Basdevant, "Evolution of high Reynolds number two-dimensional turbulence," J. Fluid Mech. 75, 305 (1975).

${ }^{38}$ J.-C. André and M. Lesieur, "Influence of helicity on the evolution of isotropic turbulence at high Reynolds number," J. Fluid Mech. 81, 187 (1977).

${ }^{39}$ D. McComb, The Physics of Fluid Turbulence (Oxford University Press, Oxford, 1992).

${ }^{40}$ T. von Kármán, "Progress in the statistical theory of turbulence," Proc. Natl. Acad. Sci. 34, 530 (1948).

${ }^{41}$ R. Zimmermann, H. Xu, Y. Gasteuil, M. Bourgoin, R. Volk, J. F. Pinton, and E. Bodenschatz, "The Lagrangian exploration module: An apparatus for the study of statistically homogeneous and isotropic turbulence," Rev. Sci. Instrum. 81, 055112 (2010).

${ }^{42}$ B. Favier, F. S. Godeferd, C. Cambon, A. Delache, and W. J. T. Bos, "Quasi-static magnetohydrodynamic turbulence at high Reynolds number," J. Fluid Mech. 681, 434 (2011). 